\title{
Assessment of clinical cases using ABO discrepancy index
}

\author{
Dr Ujjwal Pyakurel,, Dr Kamal Babu Thapaliya, ${ }^{2}$ Dr Sujaya Gupta, ${ }^{3}$ Dr Alka Gupta,4 Dr Jyoti Dhakal5 \\ 1.4Lecturer, ${ }^{5}$ Assistant Professor Dept of Orthodontics, Kantipur Dental College \\ 2Dental Surgeon, Private Practitioner, 3Lecturer, Dept of Periodontics, Kantipur Dental College
}

\section{ABSTRACT}

Introduction: The American Board of Orthodontics objectively quantifies the complexity of malocclusion before orthodontic treatment. This study aims to assess the complexity of cases as measured by ABO discrepancy index (DI) in the patients under treatment by the orthodontics residents of Kantipur Dental College (KDC). Additional objectives were to 1) Ascertain DI relative to sex, age and race/ethnicity, and 2) Differential analysis of the components of the DI.

Materials \& Method: DI was determined for 220 consecutive cases started by orthodontic residents of KDC in a three-year graduate orthodontics program from 2014-2018. The DI was scored and compared with the patient's sex and age.

Result: The DI is not statistically significant to age, sex and race/ ethnicity. The mean DI score $( \pm$ SD) was 18.65 ( \pm 10.521 ). Differential analysis of the components of the DI showed that the highest scores were for cephalometric measures, followed by overjet, crowding, occlusion, and the lowest scores were for lingual posterior crossbite.

Conclusion: The DI was a relatively reliable index for measuring malocclusion severity. It is independent of patient's age and race/ethnicity but is dependent on sex. Area of possible future improvements includes malocclusion sub-categories (Class II div. 1 and 2), and scores for bony and soft tissue impactions.

Keywords: Discrepancy index, Case complexity, Cephalometrics, Malocclusion, Orthodontic patients

\section{INTRODUCTION}

The success of the case depends upon the precise case history, diagnosis and treatment planning. Assessment of the complexity of the case is the gold standard for the optimized results. Case difficulty should be assessed which can often be subjective; however, it is related to case complexity, which can be quantifiable. Discrepancy Index (DI) has been designed by American Board of Orthodontics ( $A B O$ ) to provide an objective evaluation of the complexity of a malocclusion. This might lead to a better understanding of difficulty before starting the orthodontic treatment, which improves the compliance of the patient.

The DI is an objective method which is based on the observations and measurements taken from standard pretreatment orthodontic records i.e. study model, lateral cephalogram and panoramic radiographs.' It includes the evaluations of overjet, overbite, anterior open bite, lateral open bite, crowding, occlusion, lingual posterior crossbite, buccal posterior crossbite, and cephalometric angles i.e. Maxillo-mandibular relationship (ANB), Mandibular plane angle in relation to cranial base (SN-GoGn) and Lower incisor to Mandibular plane angle (IMPA). ${ }^{2}$ The greater the number of these conditions in a patient, the greater severity of the malocclusion and the greater the clinical effort required to achieve optimal treatment., 3

To the researcher's knowledge, no studies in Nepal have quantified the overall DI score of the patients who were treated or are being treated by the orthodontic residents. The purpose of this study was to assess the complexity of cases as measured by $\mathrm{ABO}$ discrepancy index (DI) in the patients under treatment by the orthodontic residents of Kantipur Dental College (KDC). Additional objectives were to 1) Ascertain DI relative to sex, age and race/ethnicity, and 2) Differential analysis of the components of the DI.

\section{MATERIALS AND METHOD}

Institutional review committee approval was obtained from IRC-Kantipur Dental College. This is a retrospective study conducted in Kantipur Dental College from 20142018. 220 consecutive cases were taken as a sample from the patient records that were started by orthodontic 
residents of KDC in a three-year graduate orthodontics program. Data were collected and analyzed between October and November 2018.

The criteria for inclusion were:

i. Orthodontic patients treated by the residents in the Department of Orthodontics, KDC.

ii. Natural born ethnic Nepalese. The two ethnic groups (Aryans and Mongoloids) in this study were defined as per the study of Sharma et al. ${ }^{4}$

Cases were excluded if the records were incomplete, casts were broken/damaged or the radiographs were unclear. A total of 158 cases were analyzed. Sample size of 156 was calculated using data from the study of Schafer et $a{ }^{3}$ using formula

$$
\mathrm{n}=\frac{\mathrm{Z}_{\mathrm{a}}{ }^{2} \mathrm{~s}^{2}}{\mathrm{~d}^{2}}
$$

Where,

$\mathrm{n}=$ required sample size

$\mathrm{Z}_{\mathrm{a}}=\mathrm{z}$ deviate corresponding to desired reliability level

(at 95\%, 1.96)

$\mathrm{s}=$ variance $(\mathrm{s}=$ standard deviation)

$d=$ Maximum tolerance error

Discrepancy Index scores were collected by the investigators. Casts were analyzed for overjet, overbite, anterior open bite, lateral open bite, crowding, occlusion, lingual posterior crossbite, buccal posterior crossbite, and others. Pretreatment lateral cephalometric tracings were done for ANB, SN-GoGn, and IMPA angles, Panoramic radiographs were assessed for impactions, supernumerary teeth, axial inclinations, etcand extraoral and intraoral photographs were used to collect the DI score. Scores were recorded as per the guidelines of ABO Discrepancy Index scoring system and worksheet. 5.6 Pre-treatment age, sex and race/ethnicity were also recorded from the case records of the orthodontic residents. 30 cases were scored twice, 2 weeks apart to determine the inter-examiner repeatability $(k=0.89)$.

\section{Statistical analysis}

A Spearman correlation coefficient was calculated to evaluate the association between the patient's pretreatment age with the DI scores. Mann-Whitney tests were used to evaluate the associations of the patient's sex and race/ethnicity with the DI scores. KruskalWallis Test was used to evaluate the relation between malocclusion classes and DI scores.

\section{RESULT}

An outlier, a score of 117 (next highest score, 57) was identified in the DI scores. No investigator recalled any other patient having a DI exceeding 100, so the outlier was excluded from further analysis or presentation in this report. The mean DI score ( \pm SD) was 18.65 ( \pm 10.521 ). Differential analysis of the components of the DI showed that the highest scores were for cephalometric measures, followed by overjet, crowding, occlusion, and the lowest scores were for lingual posterior crossbite. All variables are listed in Table I.

Of the 158 patients, 95 (60.1\%) were female. Average Dl scores were 17.04 9.955 for female patients and $21.06 \pm 10.962$ for male patients; these were statistically significant ( $P=0.011)$. Patients were aged $18.737 \pm 5.9608$ years (range, 8-39 years) at the start of treatment. Age at the start of treatment was not

Table 1: DI Variables

\begin{tabular}{|l|c|c|c|c|c|}
\hline \multicolumn{1}{|c|}{ Variables } & $\mathbf{n}$ & Mean \pm SD & Std. Error of Mean & Minimum & Maximum \\
\hline Overjet & 158 & $2.95 \pm 3.140$ & 0.250 & 0 & 24 \\
\hline Overbite & 158 & $1.35 \pm 1.489$ & 0.118 & 0 & 6 \\
\hline Anterior open bite & 158 & $0.80 \pm 3.195$ & 0.254 & 0 & 24 \\
\hline Lateral open bite & 158 & $0.27 \pm 1.614$ & 0.128 & 0 & 14 \\
\hline Crowding & 158 & $2.61 \pm 2.573$ & 0.205 & 0 & 12 \\
\hline Occlusion & 158 & $2.50 \pm 3.186$ & 0.253 & 0 & 4 \\
\hline Lingual posterior crossbite & 158 & $0.15 \pm 0.551$ & 0.044 & 0 & 4 \\
\hline Buccal posterior crossbite & 158 & $0.19 \pm 0.741$ & 0.059 & 0 & 43 \\
\hline Cephalometric angles & 158 & $6.01 \pm 7.079$ & 0.563 & 0 & 10 \\
\hline \multicolumn{1}{|c|}{ ANB } & 158 & $1.71 \pm 2.594$ & 0.206 & 0 & 16 \\
\hline \multicolumn{1}{|c|}{ SN-MP } & 158 & $1.72 \pm 3.293$ & 0.262 & 0 & 34 \\
\hline \multicolumn{1}{|c|}{ LI-MP } & 158 & $2.58 \pm 5.059$ & 0.402 & 0 & 22 \\
\hline Others & 158 & $1.82 \pm 3.125$ & 0.249 & & 0 \\
\hline DI Total & 158 & $18.65 \pm 10.521$ & 0.837 & & \\
\hline
\end{tabular}


Table 2: Demographics

\begin{tabular}{|c|c|c|c|c|c|c|}
\hline Variables & n (\%) & Mean \pm SD & Std. Error Mean & Minimum & Maximum & $P$ value \\
\hline Age & 158 (100\%) & $18.737 \pm 5.9608$ & 0.4742 & 8 & 39 & \multirow{4}{*}{0.908} \\
\hline$<14$ & $41(25.9 \%)$ & & & & & \\
\hline $15-25$ & $98(62 \%)$ & & & & & \\
\hline $26+$ & 19 (12\%) & & & & & \\
\hline \multicolumn{6}{|l|}{ Sex } & \multirow{3}{*}{0.011} \\
\hline Male & 63 (39.9\%) & $21.06 \pm 10.962$ & 1.381 & & & \\
\hline Female & 95 (60.1\%) & $17.04 \pm 9.955$ & 1.021 & & & \\
\hline \multicolumn{6}{|l|}{ Race/Ethnicity } & \multirow{3}{*}{0.117} \\
\hline Aryan & 111 (70.3\%) & $19.14 \pm 10.085$ & 0.957 & & & \\
\hline Mongolian & $47 \quad(29.7 \%)$ & $17.49 \pm 11.517$ & 1.680 & & & \\
\hline \multicolumn{6}{|l|}{ Malocclusions } & \multirow{4}{*}{$>0.001$} \\
\hline Class I & 71 (44.9\%) & $15.41 \pm 9.597$ & & & & \\
\hline Class II & 65 (41.1\%) & $19.86 \pm 9.359$ & & & & \\
\hline Class III & 22 (13.9\%) & $25.50 \pm 12.820$ & & & & \\
\hline Extraction & 60 (38\%) & $19.20 \pm 7.517$ & & & & \multirow{5}{*}{0.106} \\
\hline 4 premolars & 40 (25.3\%) & & & & & \\
\hline 2 maxillary premolars & $11(7 \%)$ & & & & & \\
\hline Others & $9(5.7 \%)$ & & & & & \\
\hline Non-extraction & 98 (62\%) & $18.31 \pm 12.020$ & & & & \\
\hline
\end{tabular}

significantly associated with the DI. Patients were also categorized on the basis of eruption of permanent teeth (Table 2) ${ }^{7.8}$

The population had 111 Aryans (70.3\%) and 47 Mongolians (29.7\%). Average DI scores were $19.14 \pm$ 10.085 and $17.49 \pm 11.517$ for Aryans and Mongolians respectively. These scores were not statistically significant $(P=0.117)$.

Of the total sample, most had Angle's Class I malocclusion (44.9\%), followed by Class II (41.1\%) and Class III (13.9\%). The mean DI scores were $15.41 \pm 9.597$, $19.86 \pm 9.359$ and $25.50 \pm 12.820$ for patients with Angle's
Class I, Class II and Class III malocclusions respectively. These scores were statistically significant. $(P=>0.001)$

Among 158 patients, 98 were treated without undergoing extractions, while 60 had undergone extraction/s of tooth/teeth (Table 3). Patients undergoing extraction/s had mean DI score of 19.20 \pm 7.517 , and the patients treated without extractions had mean DI score of $18.31 \pm 12.020$. The association between extractions/non-extractions with the DI score was statistically insignificant $(P=0.106)$. Among the extraction cases, majority had undergone extractions of both maxillary and mandibular premolars (25.3\%),

Table 3: DI Other Variables

\begin{tabular}{|l|c|c|c|c|c|}
\hline \multicolumn{1}{|c|}{ Others } & N & Mean \pm SD & Std. Error of Mean & Minimum & Maximum \\
\hline Supernumerary teeth & 158 & $0.04 \pm 0.306$ & 0.024 & 0 & 3 \\
\hline Ankylosis of permanent teeth & 158 & $0 \pm 0$ & 0 & 0 & 0 \\
\hline Anomalous morphology & 158 & $0.09 \pm 0.569$ & 0.045 & 0 & 10 \\
\hline Impaction (except 3rd molars) & 158 & $0.29 \pm 1.191$ & 0.095 & 0 & 2 \\
\hline Midline discrepancy ( $\geq 3 \mathrm{~mm}$ ) & 158 & $0.14 \pm 0.511$ & 0.041 & 0 & 16 \\
\hline Missing teeth (except 3rd molars) & 158 & $0.35 \pm 1.666$ & 0.133 & 0 & 14 \\
\hline Missing teeth, congenital & 158 & $0.14 \pm 1.197$ & 0.095 & 0 & 4 \\
\hline Spacing (4 or more, per arch) & 158 & $0.37 \pm 0.926$ & 0.074 & 0 & 2 \\
\hline Spacing (max. central diastema $\geq 2 \mathrm{~mm}$ ) & 158 & $0.10 \pm 0.494$ & 0.039 & 0 & 2 \\
\hline Tooth transposition & 158 & $0.03 \pm 0.224$ & 0.018 & 0 & 0 \\
\hline Skeletal asymmetry (nonsurgical tx) & 158 & $0 \pm 0$ & 0.068 & 0 & 4 \\
\hline Additional treatment complexities & 158 & $0.28 \pm 0.859$ & & 0 & 0 \\
\hline
\end{tabular}


followed by maxillary premolars only (7\%) and others (5.7\%).

\section{DISCUSSION}

We assessed the relationship of the DI to the patient's age, sex and race/ethnicity at the beginning of treatment. Statistically, there was no effect of age and race/ethnicity on the overall DI when the patients were sampled over 5 years (2014-2018). However, there was a significant difference between the DI scores of male and female patients.

In comparison, Schafer et $a^{3}$ reported an insignificant differences in DI scores for male and female patients. Male $(n=291)$ had a similar DI sore of $15.8 \pm 9.7$, compared with $15.6 \pm 11.1$ for females $(n=425)$. As expected, female patients comprised $60.1 \%$ of our sample, demonstrating that they are more likely to seek orthodontic treatment.

Urtane et $a^{9}$ reported that the severity of malocclusion and the subsequent need of orthodontic treatment increased with age. Statistically significant difference between the mean The Index of Complexity, Outcome and Need (ICON) values in all age groups (12-13, 18 and 35-44 years old) was observed. Similarly, ICON scores were determined to be higher in the age group of 18-year-olds. Further, the ICON weighs heavily on esthetics, which is highly subjective rather than objective..$^{10}$ Koochek et $a^{11}$ reported that even though the ICON showed good sensitivity for identifying treatment need, its specificity was poor. In this study, the mean pre-treatment age was about 19 years. There was no statistically significant correlation between the DI and pre-treatment age, indicating that malocclusion complexity, as measured by the Dl, does not depend on age. Pre-treatment age in this study was also significantly greater than other similar studies that evaluated graduate orthodontic programs. The mean pretreatment age at the University of Tennessee, Lovisiana State University, and the University of Alabama was reported 12.92 years. ${ }^{12}$ Similarly, YangPowers et $a^{13}$ reported a mean pre-treatment age of 14.3 years at the University of Illinois at Chicago.

An important result of this study was the finding that the DI is independent of two major Nepalese ethnic groups, ${ }^{4}$ documented by a mean DI for Aryan patients of 19.14 compared with 17.49 for Mongolian patients; this was not significantly different $(p=0.117)$.
$D$ I score seemed to be statistically insignificant $(\mathrm{p}=$ 0.106) when compared to the cases treated with or without extractions. The mean DI scores of the patients treated with or without extraction/s were 19.20 and 18.31 respectively. This implies that the initial DI score does not necessarily dictate whether the patient is going to be treated with or without extraction.

Further, the mean DI score increased with the increase in the class of malocclusion. Angle's Class I, Class II and Class III had the DI scores of 15.41, 19.86 and 25.50 respectively. This means that the severity of malocclusion as measured by the discrepancy index is highest for Class III, followed by Class II and Class I. Among the samples, $44.9 \%$ had Class I, $41.1 \%$ had Class II and $13.9 \%$ had Class III malocclusion. The number of patients having Angle Class I and Class II malocclusions were similar. In contrast, in the similar set of samples, Shrestha et $\mathrm{al}^{14}$ reported that the majority of the patients had Class I (54.70\%), followed by Class II (36.90\%) and Class III (8.40\%) which were in accordance with the results of this study.

Some of the patients having multiple bony impactions, who were treated with great difficulties for a longer period of time, had lower overall DI scores. This could mislead the case as an easy one, which pointed towards its inconsistency in assessing the complexity of the certain cases.

\section{CONCLUSION}

DI was found to be independent of age and Nepalese race/ethnicity but was dependent on sex. Males were found to have mean DI scores significantly higher than females.

DI was found to be relatively reliable index compared to previous indices to access the severity of malocclusion. But the areas of possible future improvement in the current $\mathrm{ABO} \mathrm{DI}$ could be the addition of malocclusion sub-categories (e.g. Class II Division 1 and Division 2), bony and soft tissue impactions scores modification.

\section{ACKNOWLEDGEMENT}

We thank Dr. Pooja Shah, Dr. Asal Acharya, Dr. Anju Agarwal, Dr. Diwash Adhikari, and Dr. Sanzee Silwal for their contributions in data collection. 


\section{REFERENCES}

1. Cangialosi T, Riolo M, Owens S, Dykhouse V, Moffitt A, Grubb J, Greco P, English J, James R. The ABO discrepancy index: A measure of case complexity. Am J Orthod Dentofacial Orthop. 2004;125(3):270-8.

2. Riolo M, Owens S, Dykhouse V, Moffitt A, Grubb J, Greco P, English J, Briss B, Cangialosi T. ABO resident clinical outcomes study: Case complexity as measured by the discrepancy index. Am J Orthod Dentofacial Orthop. 2005;127(2):161-3.

3. Schafer SM, Maupome G, Eckert GJ, Roberts WE. Discrepancy index relative to age, sex, and the probability of completing treatment by one resident in a 2-year graduate orthodontics program. Am J Orthod Dentofacial Orthop. 201 1;139(1):70-3.

4. Sharma JN. Steiner's cephalometric norms for the Nepalese population. JO. 2011;38:21-31.

5. $A B O$ Discrepancy Index Scoring System

6. $A B O$ Discrepancy Index Worksheet

7. Singh K, Gorea RK, Bharti V. Age estimation from eruption of permanent teeth. JIAFM. 2005;27(4):231-5.

8. Manjunatha BS, Soni NK. Estimation of age from development and eruption of teeth. J Forensic Dent Sci. 2014;6(2):73-6.

9. Urtane I, Pugaca J, Liepa A, Rogovska I. The severity of malocclusion and need for orthodontic treatment in correspondence with the age. Stomatologija. 2006;8(2):35-8.

10. Llewellyn SK, Hamdan AM, Rock WP. An index of orthodontic treatment complexity. Eur J Orthod. 2007;29(2):186-92.

11. Koochek A, Yeh MS, Rolfe B, Richmond S. The relationship between index of complexity, outcome and need, and patients' perceptions of malocclusion: a study in general dental practice. Br Dent J. 2001;191 (6):325-9.

12. Cook DR, Harris EF, Vaden JL. Comparison of university and private-practice orthodontic treatment outcomes with the American Board of Orthodontics objective grading system. Am J Orthod Dentofacial Orthop. 2005;127(6):707-12.

13. Yang-Powers LC, Sadowsky C, Rosenstein S, BeGole EA. Treatment outcome in a graduate orthodontic clinic using the American Board of Orthodontics grading system. Am J Orthod Dentofacial Orthop. 2002;122(5):451-5.

14. Shrestha S, Shrestha RM. An analysis of malocclusion and occlusal characteristics in Nepalese orthodontic patients. Orthodontic Journal of Nepal. 2013;3(1):19-25.

15. Parrish L, Roberts WE, Maupome G, Stewart K, Bandy RW, Kula KS. The relationship between the ABO discrepancy index and treatment duration in a graduate orthodontic clinic. Angle Orthod. 2011;81(2):192-7.

16. Campbell CL, Roberts WE, Hartsfield J, Qi R. Treatment outcomes in a graduate orthodontic clinic for cases defined by the American Board of Orthodontics malocclusion categories. Am J Orthod Dentofacial Orthop. 2007;132(6):822-9

17. Knierim K, Roberts WE, Hartsfield J. Assessing treatment outcomes for a graduate orthodontics program: Follow-up study for the classes of 2001-2003. Am J Orthod Dentofacial Orthop. 2006;130(5):648-55. 